\title{
BMJ Open Predicting mortality in acute ischaemic stroke treated with mechanical thrombectomy: analysis of a multicentre prospective registry
}

\author{
Hao Li, ${ }^{1}$ Shi-sheng Ye (D) , ${ }^{1}$ Yuan-Ling Wu, ${ }^{2}$ Sheng-Ming Huang, ${ }^{3}$ Yong-Xin Li, ${ }^{4}$ \\ Kui Lu, ${ }^{5}$ Jing-Bo Huang, ${ }^{1}$ Lve Chen, ${ }^{4}$ Hong-Zhuang Li, ${ }^{4}$ Wen-Jun Wu, ${ }^{5}$ Zhi-Lin Wu, ${ }^{6}$ \\ Jian-Zhou Wu, ${ }^{6}$ Wang-Tao Zhong, ${ }^{7}$ Wen-Chuan Xian, ${ }^{7}$ Feng Liao, ${ }^{7}$ \\ Tao-Hsin Tung (D) , ${ }^{8}$ Qiao-Ling Wu, ${ }^{1}$ Hai Chen, ${ }^{1}$ Li Yuan, ${ }^{1}$ Zhi Yang, ${ }^{1}$ Li-An Huang ${ }^{3}$
}

To cite: Li H, Ye S, Wu Y-L, et al. Predicting mortality in acute ischaemic stroke treated with mechanical thrombectomy: analysis of a multicentre prospective registry. BMJ Open 2021;11:e043415. doi:10.1136/ bmjopen-2020-043415

- Prepublication history and supplemental material is published online only. To view please visit the journal online (http://dx.doi.org/10.1136/ bmjopen-2020-043415).

$\mathrm{HL}$ and S-sY contributed equally.

Received 20 August 2020 Revised 09 November 2020 Accepted 02 February 2021

Check for updates

(c) Author(s) (or their employer(s)) 2021. Re-use permitted under CC BY-NC. No commercial re-use. See rights and permissions. Published by BMJ.

For numbered affiliations see end of article.

Correspondence to Dr Zhi Yang; mmyangz@163.com and Dr Li-An Huang;

huanglian1306@126.com

\section{ABSTRACT}

Objectives We aimed to determine predictors of mortality within 90 days and develop a simple score for patients with mechanical thrombectomy (MT).

Design Analysis of a multicentre prospective registry. Setting In six participating centres, patients who had an acute ischaemic stroke (AIS) treated by MT between March 2017 and May 2018 were documented prospectively. Participants 224 patients with AIS were treated by MT. Results of 224 patients, 49 (21.9\%) patients died, and $87(38.8 \%)$ were independent. Variables associated with 90-day mortality were age, previous stroke, admission National Institutes of Health Stroke Scale (NIHSS), fasting blood glucose and occlusion site. Logistic regression identified four variables independently associated with 90-day mortality: age $\geq 80$ years (OR $3.26,95 \% \mathrm{Cl} 1.45$ to 7.33 ), previous stroke (OR $2.33,95 \% \mathrm{Cl} 1.04$ to 5.21 ), admission NIHSS $\geq 18$ (OR 2.37, 95\% $\mathrm{Cl} 1.13$ to 4.99 ) and internal carotid artery or basilar artery occlusion (OR 2.92, $95 \% \mathrm{Cl} 1.34$ to 6.40 ). Using these data, we developed predicting 90-day mortality of AIS with MT (PRACTICE) score ranging from 0 to 6 points. The receiver operator curve analysis found that PRACTICE score (area under the curve $(\mathrm{AUC})=0.744,95 \% \mathrm{Cl} 0.669$ to 0.820 ) was numerically better than iScore (AUC $=0.661,95 \% \mathrm{Cl} 0.577$ to 0.745 ) and Predicting Early Mortality of Ischemic Stroke score (AUC=0.638, 95\% Cl 0.551 to 0.725 ) for predicting 90-day mortality.

Conclusions We developed a simple score to estimate the 90-day mortality of patients who had an AIS treated with MT. But the score needs to be prospectively validated. Trial registration number Chinese Clinical Trial Registry (ChiCTR-00C-17013052).

\section{INTRODUCTION}

Previous randomised controlled trials have demonstrated the overwhelming benefit of mechanical thrombectomy (MT) for acute ischaemic stroke (AIS) due to emergent large vessel occlusion. ${ }^{1-6}$ Although patients undergo MT as first-line therapy, the mortality within 90 days remains high: more than $30 \%$
Strengths and limitations of this study

- The study used multicentre prospective data from consecutively admitted patients who had an ischaemic stroke treated with mechanical thrombectomy.

- Multivariate logistic regression analysis only used variables before the procedure, predicting 90-day mortality before mechanical thrombectomy.

- The PRACTICE (Predicting 90-days mortality of AIS with MT) score was only validated in the primary data set, and future external validation should be performed in another data set.

- The study was based on a retrospective analysis of a prospective observational registry.

in patients with acute basilar artery occlusion and approximately $15 \%$ in patients with large-vessel occlusion in the anterior circulation. ${ }^{67}$ Previous studies have found predictors associated with mortality in patients who had an ischaemic stroke, such as age, diabetes mellitus, National Institutes of Health Stroke Scale (NIHSS) score, vessel occlusion site, increased diffusion weighted imaging (DWI) lesional volume, passes with the thrombectomy device and use of rescue therapy. ${ }^{7-11}$ The ability to predict mortality from information available prior to MT could be informative for patients and their family members. Such knowledge would impact on family members decision-making before MT and neurologists management decisions as well as medical care after MT. Unfortunately, a prognostic scoring system to predict mortality in patients undergoing MT has been less intensively addressed thus far. Many prognostic scoring systems, such as Houston Intra-Arterial recanalisation Therapy (HIAT) score, HIAT2 score, Stanford Age and DWI (SAD) score and the Totaled Health Risks in Vascular Events (THRIVE) 
score were developed to predict poor outcome, ${ }^{8} 11-13$ but not for mortality specially. The iScore and PLAN (Preadmission comorbidities, Level of consciousness, Age, and focal Neurologic deficit) score were developed for mortality prediction in AIS. ${ }^{14}{ }^{15}$ However, iScore and PLAN score were validated to predict outcomes in patients undergoing endovascular therapy due to anterior circulation large artery occlusion, showing moderate predictive accuracy (iScore area under the curve (AUC) $=0.76$ and PLAN score AUC=0.73) ${ }^{16}$ Recently, the Predicting Early Mortality of Ischemic Stroke (PREMISE) score was developed to estimate early mortality of patients who had an ischaemic stroke treated at a stroke unit with good predictive accuracy $(\mathrm{AUC}=0.879) .{ }^{10}$ However, PREMISE score derived from nationwide Austrian Stroke Unit Registry, including a relatively small patients treated with MT, and the validation of PREMISE score in thrombectomy cohort has not been reported yet.

Overall, the prognostic scoring system to predict mortality of patients with MT remain lacking, and it is critical to avoid futile MT by predicting mortality from clinical and imaging information available prior to MT. Our study aimed to determine predictors of mortality within 90 days, develop a simple scoring instrument for patients with MT and compared the predictive power with the previous prognostic tools (iScore, PREMISE score) in a multicentre prospective registry study for endovascular treatment of AIS (ET-AIS).

\section{METHOD}

\section{Patients selection}

Three hundred and three consecutive patients presenting with AIS treated by endovascular treatment were registered in the ET-AIS registry during March 2017 and May 2018. ET-AIS was a multicentre, prospective and observational registry study involving six comprehensive stroke centres in China. In our study, six comprehensive stroke centres were divided into low volume centre (Zhongshan People's Hospital, Yunfu People's Hospital, Affiliated Hospital of Guangdong Medical University), medium volume centre (First Affiliated Hospital of Jinan University, Shunde Hospital of Southern Medical University) and high volume centre (Maoming People's Hospital) according to the number of patients included in ET-AIS registry. Low, medium and high volume centre were defined as number of included patients $<50,50 \leq$ included patients $<100$ and included patients $\geq 100$. Patients receiving intravenous thrombolysis (IVT) prior to ET were acceptable, consistent with current guidelines. ${ }^{17}$

All consecutive patients with the following criteria were included: (1) age 18 years or older; (2) diagnosis of an AIS with proven large vessel occlusion confirmed by digital subtraction angiography; (3) treatment with or without IVT prior to MT; and (4) treated with MT within 12 hours of symptom onset. Patients with prestroke functional dependence (modified Rankin Scale (MRS) score $>2$ ), only intracranial large vascular stenosis, only anterior cerebral artery (ACA) occlusion, only posterior cerebral artery (PCA) or vertebral artery (VA) occlusion were excluded.

\section{Study variables and outcome definition}

All data, including baseline characteristics, procedure details and outcome, were recorded on case report forms. Patient demographics included age and gender. The main vascular risk factors included smoking (defined as self-reports of cigarette smoking in the past, or currently), hypertension, diabetes mellitus, coronary artery disease, atrial fibrillation and previous stroke. A history of hypertension, diabetes mellitus, coronary artery disease and atrial fibrillation was based on documentation at admission and did not include a new diagnosis made during incident hospitalisation. Laboratorial tests included admission fasting plasma glucose (FPG) and admission low-density lipoprotein cholesterol. Clinical and radiological data included the admission NIHSS score, Alberta Stroke Program Early CT Score (ASPECTS) on non-contrast CT, occlusion site, Trial of ORG 10172 in the Acute Stroke Treatment classification, preprocedure modified Thrombolysis in Cerebral Infarction (m-TICI) score and time from onset to groin puncture. Procedure details included IVT prior to the procedure, methods of anaesthesia, stenting (extracranial stenting and/or intracranial stenting) and procedure-related complications. Outcomes included successful recanalisation (defined as postprocedure m-TICI $\geq 2 \mathrm{~b}$ ), intracranial haemorrhage within 24 hours post-MT, 90-day favourable outcome (defined as MRS $\leq 2$ ) and 90-day mortality (defined as MRS=6). Intracranial haemorrhage (ICH) was defined by the Heidelberg Bleeding Classification and classified into symptomatic ICH (SICH) and asymptomatic ICH (aSICH). ${ }^{18}$ SICH confirmed by the 24-hour follow-up head CT or MRI with a $\geq 4$ point NIHSS score increase according to the Heidelberg criteria. A follow-up was performed at 90 days by neurological physicians or trained research nurses during face-to-face interviews or via telephone conversations with the patient, their relatives or their general practitioner. More details of variables and outcomes definition are shown in online supplemental material.

\section{Endovascular procedures}

Before entry into the neuroangiography suite, all patients or legally authorised representatives signed informed consent forms. All procedures were performed under general anaesthesia or conscious sedation. The major revascularisation method was MT using a stent retriever or direct aspiration at the operator's discretion. If underlying intracranial atherosclerotic stenosis was detected, balloon angioplasty or stenting was performed when considered necessary. Intra-arterial thrombolysis was allowed as adjuvant therapy for some patients. Recanalisation status was quantified by using the m-TICI score based on the final angiogram, and successful recanalisation was defined as an m-TICI score $\geq 2 \mathrm{~b} .{ }^{19}$ All patients underwent 
head CT scan or MRI within 24 hours after the procedure to assess haemorrhagic complications. Specific endovascular procedures and preoperative anaesthesia methods were chosen by operators at each centre. For postoperative antithrombotic drugs, antiplatelet drugs or anticoagulants were selected depending on the stroke aetiology.

\section{Calculation of iScore and PREMISE score}

The iScore has 30-day and 1-year versions, and we use the 30 -day version in this study, which derived from age (1 point for per year), sex (10 points for men), stroke severity (105 points for Canadian Neurological Scale $(\mathrm{CNS})=0,65$ points for $\mathrm{CNS} \leq 4,40$ points for $\mathrm{CNS}=5-7$ and 0 point for CNS $\geq 8$ ), stroke subtype ( 0 point for lacunar, 30 points for non-lacunar and 35 points for undetermined origin), risk factor (10 points for atrial fibrillation and congestive heart failure, respectively), comorbid condition (10 points for cancer and 35 points for renal dialysis), preadmission disability (15 points for dependent) and glucose on admission (15 points for $\geq 135 \mathrm{mg} / \mathrm{dL}$ ). ${ }^{14} \mathrm{In}$ ET-AIS registry, stroke severity was assessed on admission by using the NIHSS, which could be converted to CNS score according to the following conversion: a CNS score of 1-4 equals an NIHSS score of 14-22, a CNS score of 5-7 equals an NIHSS score of 9-13, a CNS score of $\geq 8$ equals an NIHSS score of $\leq 8$ and a CNS score of 0 equals an NIHSS score of $>22 .^{20}$

The PREMISE score with a total score of 12 was calculated on the basis of age ( 1 point for 60-69 years and 2 points for $\geq 70$ years), stroke severity ( 2 points for NIHSS score $5-11,4$ points for NIHSS score 12-23, 5 points for NIHSS score $\geq 24$ ), prestroke functional disability ( 1 point for MRS scores 1-5), pre-existing heart disease (1 point for yes), diabetes mellitus (1 point for yes), posterior circulation stroke syndrome (1 point for yes) and nonlacunar stroke cause ( 1 point for yes). ${ }^{10}$

\section{Statistical analysis and score development}

Statistical analyses were performed using SPSS V.25.0. A $\mathrm{p}$ value $<0.05$ was considered statistically significant (two-sided). Continuous variables are reported as the means $\pm \mathrm{SD}$ or medians (IQR), and categorical variables are reported as frequencies. Comparisons were performed on mortality and survival using the Pearson $\chi^{2}$ test, independent-samples t-test and Mann-Whitney U test. To account for multiple testing, the significance level was set at $\mathrm{p}<0.05$. Multivariate logistic regression analysis was performed to determine independent predictors for 90-day mortality. We tested the association of procedurerelated variables with mortality; however, because the primary goal of the study was predicting mortality before the procedure, these variables were not used in the multivariate model.

The PRACTICE (Predicting 90-days mortality of AIS with MT) score was developed based on variables that were independently associated with 90-day mortality in multivariate analysis. By rounding to the next positive integer value, the score point could be defined according to the value of the $\beta$-coefficient, and the total risk score could be calculated by summing all the components of the score. The threshold values of the PRACTICE score were determined by using a receiver operating characteristic (ROC) curve and its sensitivity and specificity to evaluate the authenticity and accuracy of the PRACTICE score. We also validated the iScore and PREMISE score in our registry, comparing the predictive power with PRACTICE score. Model calibration was assessed using the Hosmer-Lemeshow goodness-of-fit test.

\section{Patient and public involvement}

Patients and/or the public were not involved in the design, or conduct, or reporting, or dissemination plans of this research.

\section{RESULTS}

During the study period, a total of 303 patients with AIS were treated by endovascular recanalisation in six participating centres. Of these, 79 patients were excluded from the present study analysis for the following reasons: lost to follow-up at 90 days $(n=12)$, only stenosis $(n=54)$, ACA occlusion $(n=5)$, PCA occlusion $(n=3)$ and VA occlusion $(n=5)$. A total of 224 patients finally met the study inclusion criteria. The mean age was $67.08 \pm 12.94$ years, and $39.7 \%$ were women. Procedure-related complications occurred in 77 patients $(34.3 \%)$, including vasospasm $(n=33)$, stent thrombosis $(n=4)$, dissection $(n=8)$, new vessel occlusion $(n=29)$ and subarachnoid haemorrhage caused by vascular perforation $(n=3)$. ICH occurred in 25 patients, among whom $8(3.6 \%)$ were symptomatic. At the 90-day follow-up, $49(21.9 \%)$ patients had died, and $87(38.8 \%)$ were independent (90-day MRS $\leq 2)$.

The mortality group had a significantly higher prevalence of age $\geq 80$ years $(p<0.001)$, previous stroke $(\mathrm{p}=0.049)$, admission NIHSS score $\geq 18(\mathrm{p}<0.001)$, FPG $>150 \mathrm{mg} / \mathrm{dL}(\mathrm{p}=0.023)$ and internal carotid artery (ICA) or basilar artery (BA) occlusion $(p=0.001)$ than the survival group had (table 1). There was no significant difference in the use of IVT, general anaesthesia, stenting, procedure-related complications, recanalisation or ICH between the mortality group and the survival group. In a multivariate logistic regression analysis (table 2), age $\geq 80$ years (adjusted OR 3.26, 95\% CI 1.45 to 7.33 , p=0.004), previous stroke (adjusted OR 2.33, 95\% CI 1.04 to 5.21, $\mathrm{p}=0.040$ ), admission NIHSS score $\geq 18$ (adjusted OR 2.37, 95\% CI 1.13 to $4.99, \mathrm{p}=0.023$ ) and ICA or BA occlusion (adjusted OR 2.92, 95\% CI 1.34 to $6.40, \mathrm{p}=0.007$ ) remained independently associated with a higher risk of 90-day mortality. Calibration of the model was good (Hosmer-Lemeshow goodness-of-fit $\mathrm{p}=0.77$ ).

All variables positively associated with early stroke mortality in the multivariate model were subsequently used to develop the PRACTICE score, which is shown in table 3. The AUC of the PRACTICE score was 0.744 (95\% CI 0.669 to $0.820, \mathrm{p}<0.001$ ), which suggested moderate diagnostic value for the prediction of 90-day mortality 


\begin{tabular}{|c|c|c|c|}
\hline Variables & $\begin{array}{l}\text { Mortality } \\
(\mathrm{N}=49)\end{array}$ & $\begin{array}{l}\text { Survival } \\
(\mathrm{N}=175)\end{array}$ & $P$ value \\
\hline Age $\geq 80$ years, $n(\%)$ & $18(36.7)$ & $25(14.3)$ & $<0.001$ \\
\hline Male sex, n (\%) & $31(63.3)$ & $104(59.4)$ & 0.628 \\
\hline Smoking, $\mathrm{n}(\%)$ & $12(24.5)$ & $46(26.3)$ & 0.800 \\
\hline Hypertension, n (\%) & $27(55.1)$ & $80(45.7)$ & 0.245 \\
\hline Diabetes, n (\%) & $12(24.5)$ & $28(16.0)$ & 0.170 \\
\hline Atrial fibrillation, $\mathrm{n}(\%)$ & $18(36.7)$ & $54(30.9)$ & 0.436 \\
\hline $\begin{array}{l}\text { Coronary artery disease, } \\
\mathrm{n}(\%)\end{array}$ & $9(18.4)$ & $17(9.7)$ & 0.095 \\
\hline Previous stoke, n (\%) & $16(32.7)$ & $34(19.4)$ & 0.049 \\
\hline TOAST classification, n (\%) & & & 0.902 \\
\hline Large artery atherosclerosis, & $18(36.7)$ & $68(38.9)$ & \\
\hline
\end{tabular}

$$
\mathrm{n}(\%)
$$

\begin{tabular}{|c|c|c|c|}
\hline Cardioembolism, n (\%) & $21(42.9)$ & $79(45.1)$ & \\
\hline Others, n (\%) & $10(20.4)$ & $28(16.0)$ & \\
\hline $\begin{array}{l}\text { Admission NIHSS score } \geq 18 \text {, } \\
\mathrm{n}(\%)\end{array}$ & $33(67.3)$ & $66(37.7)$ & $<0.001$ \\
\hline FPG >150 mg/dL, n (\%) & $22(47.8)$ & $50(29.9)$ & 0.023 \\
\hline LDL-C >160 mg/dL, n (\%) & $12(26.7)$ & $31(18.6)$ & 0.230 \\
\hline $\begin{array}{l}\text { Time from onset to groin } \\
\text { puncture }\end{array}$ & & & 0.611 \\
\hline$<6$ hours, $\mathrm{n}(\%)$ & $27(55.1)$ & $110(62.9)$ & \\
\hline 6-12 hours, n (\%) & $19(38.8)$ & $57(32.6)$ & \\
\hline$\geq 12$ hours, $n(\%)$ & $3(6.1)$ & $8(4.6)$ & \\
\hline ASPECTS $\geq 6, n(\%)$ & $30(88.2)$ & $148(94.9)$ & 0.233 \\
\hline Occlusion site, n (\%) & & & 0.001 \\
\hline MCA, n (\%) & $13(26.5)$ & $92(52.6)$ & \\
\hline ICA/BA, n (\%) & $36(73.5)$ & $83(47.4)$ & \\
\hline Use of IVT, n (\%) & $19(38.8)$ & $67(38.3)$ & 0.950 \\
\hline $\begin{array}{l}\text { General anaesthesia, } \\
\mathrm{n}(\%)\end{array}$ & $6(12.0)$ & $27(15.4)$ & 0.143 \\
\hline $\begin{array}{l}\text { Stenting (extracranial stenting } \\
\text { and/or intracranial stenting), } \\
\mathrm{n}(\%)\end{array}$ & $21(41.2)$ & $69(38.8)$ & 0.756 \\
\hline Procedure-related complications & & & 0.605 \\
\hline Vasospasm, n (\%) & $5(10.4)$ & $28(16.1)$ & \\
\hline Stent thrombosis, n (\%) & $0(0)$ & $4(2.3)$ & \\
\hline Dissection, n (\%) & $2(4.2)$ & $6(3.4)$ & \\
\hline $\begin{array}{l}\text { Embolism in a new territory, } \mathrm{n} \\
(\%)\end{array}$ & $9(18.8)$ & $20(11.5)$ & \\
\hline $\begin{array}{l}\text { SAH caused by vascular } \\
\text { perforation, } \mathrm{n}(\%)\end{array}$ & $1(2.1)$ & $2(1.1)$ & \\
\hline $\begin{array}{l}\text { Recanalisation (postprocedure } \\
\mathrm{mTICI} \geq 2 \mathrm{~b}), \mathrm{n}(\%)\end{array}$ & $44(86.3)$ & $157(89.7)$ & 0.491 \\
\hline $\mathrm{ICH}, \mathrm{n}(\%)$ & & & 0.915 \\
\hline $\mathrm{SICH}, \mathrm{n}(\%)$ & $3(4.1)$ & $5(2.9)$ & \\
\hline aSICH, n (\%) & $6(12.2)$ & $11(6.3)$ & \\
\hline
\end{tabular}

aSICH, asymptomatic ICH; ASPECTS, Alberta Stroke Program Early CT Score; BA, basilar artery; FPG, fasting plasma glucose; ICA, internal carotid artery; ICH, intracranial haemorrhage; IVT, intravenous thrombolysis; LDL-C, low-density lipoprotein cholesterol; MCA, middle cerebral artery; mTICl, modified Thrombolysis in Cerebral Infarction; NIHSS, National Institutes of Health Stroke Scale; SAH, subarachnoid haemorrhage; $\mathrm{SICH}$, symptomatic ICH; TOAST, Trial of ORG 10172 in the Acute Stroke Treatment.
Table 2 Multivariate logistic regression model including variables associated with 90-day mortality

\begin{tabular}{llll}
\hline Variables & $\boldsymbol{\beta}$ & OR $(\mathbf{9 5 \%} \mathbf{~ C l})$ & P value \\
\hline Age $\geq 80$ years & 1.181 & $3.26(1.45$ to 7.33$)$ & 0.004 \\
Previous stoke & 0.844 & $2.33(1.04$ to 5.21$)$ & 0.040 \\
$\begin{array}{l}\text { Admission } \\
\text { NIHSS score } \geq 18\end{array}$ & 0.863 & $2.37(1.13$ to 4.99$)$ & 0.023 \\
FPG $>150 \mathrm{mg} / \mathrm{dL}$ & 0.646 & $1.91(0.92$ to 3.97$)$ & 0.084 \\
$\begin{array}{l}\text { Occlusion site } \\
\text { MCA }\end{array}$ & Reference & Reference & Reference \\
\hline ICA/BA & 1.072 & $2.92(1.34$ to 6.40$)$ & 0.007 \\
\hline
\end{tabular}

BA, basilar artery; FPG, fasting plasma glucose; ICA, internal carotid artery; MCA, middle cerebral artery; NIHSS, National Institutes of Health Stroke Scale.

(figure 1). In terms of the cut-off value, $\geq 2.5$ had a sensitivity of $69 \%$, specificity of $67 \%$, positive predictive value of $37 \%$ and negative predictive value of $89 \%$ for predicting 90-day mortality. Furthermore, PRACTICE score had better prediction power than iScore (AUC $=0.661,95 \%$ CI 0.577 to 0.745 ) and PREMISE score (AUC $=0.638,95 \%$ CI 0.551 to 0.725 ) for predicting 90 -day mortality.

\section{DISCUSSION}

In our study, we used data from a multicentre prospective registry to identify pretreatment risk factors for 90-day mortality of AIS treated with MT. The score consists of four clinical and imaging variables: age, previous stroke, admission NIHSS and occlusion site, which can explain $69 \%$ of 90-day mortality and may provide useful information in the process of decision-making before MT.

Other studies to find predictors of outcome among patients undergoing endovascular treatment have been conducted. The earliest prediction model for the poor outcome of AIS with endovascular treatment is the HIAT score developed in 2009. ${ }^{12}$ The HIAT score involves age $>75$ years, NIHSS $>18$ and admission glucose $>150 \mathrm{mg} /$ $\mathrm{dL}$, with a total score of 3 . The increasing HIAT scores revealed an increase in the rate of poor outcome on hospital discharge. The HIAT score is entirely based on clinical variables. However, there are a number of imaging factors that may correlate with outcome after endovascular

Table 3 Predicting 90-day mortality of acute ischaemic stroke with mechanical thrombectomy (PRACTICE)

\begin{tabular}{ll}
\hline Risk factors for 90-day mortality & Points \\
\hline Age $\geq 80$ years & 2 \\
Previous stoke & 1 \\
ICA/BA occlusion & 2 \\
Admission NIHSS $\geq 18$ & 1 \\
Maximal score points & 6 \\
\hline
\end{tabular}

BA, basilar artery; ICA, internal carotid artery; NIHSS, National Institutes of Health Stroke Scale. 


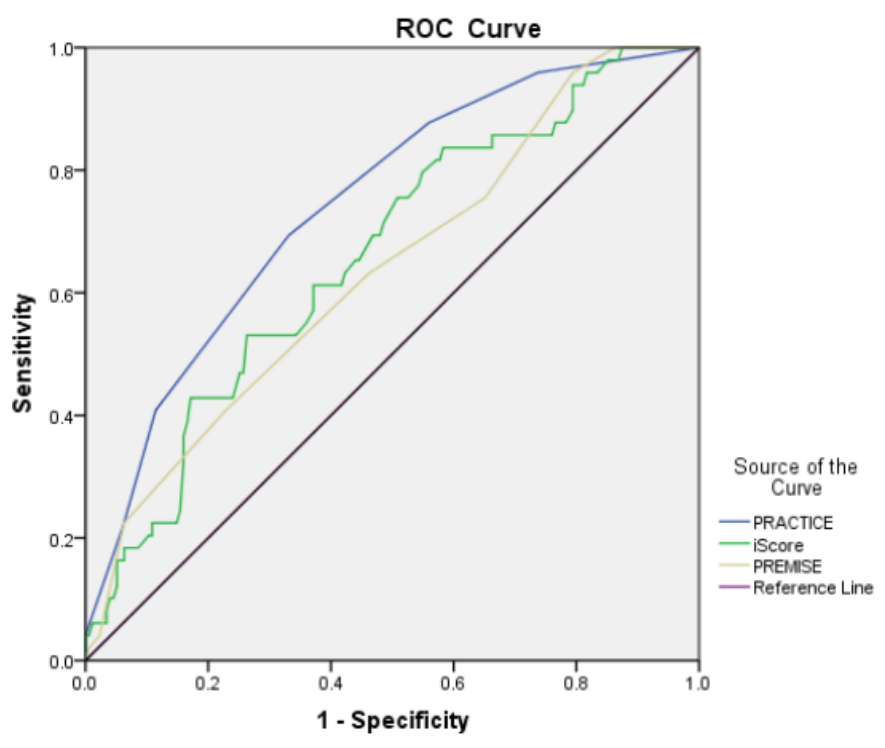

Figure 1 Comparison of PRACTICE with iScore and PREMISE score by ROC curve analysis. PRACTICE, predicting 90-days mortality of acute ischaemic stroke with mechanical thrombectomy; PREMISE, Predicting Early Mortality of Ischemic Stroke; ROC, receiver operating characteristic.

treatment. The HIAT2 score is the optimised version of the HIAT score by inclusion of ASPECTS, which more accurately predicts poor outcome after intra-arterial recanalisation therapy in anterior circulation occlusions. ${ }^{13}$ The SAD score, a score encompassing age and diffusionweighted imaging lesion volume, ${ }^{11}$ showed only a slightly better predictive accuracy than the HIAT score in the validation cohort (AUC for $\mathrm{SAD}=0.69$; HIAT $=0.66$ ). Therefore, the HIAT2 and SAD scores suggest that the addition of imaging variables to a clinical prediction scoring system may enhance the ability to predict a poor outcome following endovascular treatment. However, no model seems suitable for making individual patient decisions because of their modest AUC values. In addition, the above-mentioned score focused on poor outcome (MRS 4-6 points), not worst outcome/mortality. The THRIVE incorporate history of hypertension, history of atrial fibrillation, diabetes mellitus, age and stroke severity, and this assessment strongly predicts mortality at 90 days (ROC AUC $=0.709) .{ }^{8}$ However, the THRIVE score only consists of clinical variables and lacks imaging variables that could contribute to mortality.

The score variables of iScore and PLAN score were easyto-collect clinical parameters and comorbid conditions, which were available at the time of admission and may be determined by non-specialist clinicians. But in our registry, the detail of neurological deficit (each item of NIHSS score) was not performed systematically, so the variable were not available on all patients and PLAN score was not included in the analysis. Chu $e t a l^{16}$ validated iScore and PLAN score in AIS with anterior circulation large vessel occlusion undergoing MT in Chinese, and found that iScore is a more valid predictive tool. In our study, PRACTICE score performed better than iScore $(\mathrm{AUC}=0.744$ vs $\mathrm{AUC}=0.661)$. The PREMISE score showed a low predictive power (AUC=0.638) for predicting 90-day mortality in our study. It is possible that PREMISE score was more suitable for predicting early ( $\leq 7$ days poststroke) mortality of patients admitted with ischaemic stroke treated with medical treatment.

Our work is in agreement with previous research that has identified the occlusion site of the ICA/BA as an independent predictor of 90-day mortality. ${ }^{7}$ In that study, successfully recanalised patients had lower mortality ( $25.2 \%$ vs $46.9 \%, \mathrm{p}<0.001)$. In our study, the survival group had higher rates of successful recanalisation than the mortality group, but the difference was not statistically significant. In particular, a multiple regression analysis of the North American Solitaire Acute Stroke registry showed that occlusion sites of the ICA or BA carry a worse prognosis than middle cerebral artery occlusions despite successful recanalisation. ${ }^{21}$ Another study examined patients with acute occlusion of the distal ICA treated with intra-arterial thrombolysis or intravenous recombinant tissue plasminogen activator, and the mortality rate was $50 \%$ despite complete recanalisation. ${ }^{22}$ Although basilar occlusion accounts for approximately 5\%-10\% of all proximal intracranial occlusions, the mortality is higher than that in patients with large vessel occlusion of the anterior circulation. ${ }^{67}$ This correlation may be the result of a high lesion volume with proximal arterial occlusion, which is an independent predictor of 90-day mortality. ${ }^{11} 23$

High NIHSS on admission has previously been correlated with poor outcomes or mortality after endovascular treatment by several studies. ${ }^{37-10}$ In the present study, we determined a cut-off value of 18 , which was predictive of 90-day mortality. Patients who present with a high NIHSS have even poorer outcomes with intravenous therapy or medical management compared with endovascular treatment. ${ }^{3}$

Age has previously been associated with poor outcome in patients treated with intravenous therapy and endovascular treatment. ${ }^{10-1324}$ In our study, age $\geq 80$ years was a significant independent predictor of 90-day mortality and scored 2 points in the model. Very recently, an analysis of German Stroke Registry-Endovascular Treatment showed that patients aged $\geq 90$ years have higher mortality for thrombectomy. ${ }^{25}$ Decision-making for thrombectomy in patients aged $\geq 90$ years should be premised on a case-bycase basis with regard to admission NIHSS and ASPECT scores.

PRACTICE score has only moderate predictive power (AUC=0.74) for predicting mortality, which seems unsuitable for making decision to proceed to MT. However, the MT decision should be made individually based on clinical judgement and family decision. Until prospective data from randomised trials are available, the PRACTICE score could be useful to provide valuable prognostic data and to adjust expectations of outcome before MT. 


\section{Limitations}

This study has several limitations. First, the study was based on a retrospective analysis of a prospective observational registry, and PRACTICE score needs to be validated prospectively in another data set. Second, the imaging assessments were not adjudicated by a core laboratory, which could introduce a bias in estimates. Third, potential Berkson's bias (selection bias) was inevitable due to the hospital-based study design. This population was not only exactly representative of the whole general population, but it was also difficult to estimate the mortality of a matched total population sample in China, preferably in the region of Guangdong. Fourth, some of the clinical variables obtained from medical records could have resulted in misclassification bias. Nevertheless, it seems reasonable to assume that if misclassification bias was present, it was not related to all-cause mortality, thus could be viewed as non-differential misclassification. Finally, the small sample size limited stratified analysis of predictor for insuffcient statistical power,such as age and NIHSS score.

\section{CONCLUSION}

In summary, a prediction score based on age, previous stroke, admission NIHSS and occlusion site predicted 90-day mortality following MT with moderate accuracy. This score may provide useful information in the process of decision-making before MT. But the score needs to be prospectively validated.

\section{Author affiliations}

${ }^{1}$ Department of Neurology, Maoming People's Hospital, Maoming, China

${ }^{2}$ Department of Neurology, Longgang District People's Hospital of Shenzhen,

Shenzhen, China

${ }^{3}$ Department of Neurology, Jinan University First Affiliated Hospital, Guangzhou, China

${ }^{4}$ Department of Neurology, Shunde Hospital of Southern Medical University, Foshan, China

${ }^{5}$ Department of Neurology, Zhongshan City People's Hospital, Zhongshan, China ${ }^{6}$ Department of Integrated Intervention, Yunfu People's Hospital, Yunfu, China ${ }^{7}$ Department of Neurology, Affiliated Hospital of Guangdong Medical University, Zhanjiang, China

${ }^{8}$ Department of Enze Medical Research Center, Affiliated Taizhou Hospital of Wenzhou Medical College, Taizhou, China

Correction notice This article has been corrected since it was published. Dr Zhi Yang has been added as co-corresponding author.

Acknowledgements We thank all ET-AIS registry collaborators.

Contributors $\mathrm{ZY}$ and L-AH organised this research. HL and S-sY were mainly responsible for collecting data, data proofreading, data statistical analysis and writing the paper. All the authors participated in the initiation of the research project and the design of the research contents. The research plan and paper case report form (CRF) were written by ZY, L-AH, H-ZL, W-JW, Z-LW and W-TZ. Y-LW, S-MH, HL, $S-S Y, Y-X L, K L, J-B H, Q-L W, H C, L Y, L C, J-Z W, W-C X$ and FL were mainly responsible for the collection of cases in each centre, completion of the paper CRF and the registration of data on the electronic CRF registration website. T-HT assisted with analysis of data and manuscript revision. All authors have read and approved the final manuscript.

Funding This study was supported by Guangdong Natural Fund Project (No: 32218145), High-level Hospital Construction Research Project of Maoming People's Hospital and The Science and Technology Plan Project of Maoming (No: 2017004).
Competing interests None declared.

Patient consent for publication Not required.

Ethics approval The registry study protocol was approved by the ethics committee of First Affiliated Hospital of Jinan University (ID:2017007), ethics committee of Maoming People's Hospital, ethics committee of Shunde Hospital of Southern Medical University, ethics committee of Zhongshan People's Hospital, ethics committee of Yunfu People's Hospital and ethics committee of Affiliated Hospital of Guangdong Medical University.

Provenance and peer review Not commissioned; externally peer reviewed.

Data availability statement Data are available upon reasonable request. The data that support the findings of this study are available from the corresponding author upon reasonable request.

Supplemental material This content has been supplied by the author(s). It has not been vetted by BMJ Publishing Group Limited (BMJ) and may not have been peer-reviewed. Any opinions or recommendations discussed are solely those of the author(s) and are not endorsed by BMJ. BMJ disclaims all liability and responsibility arising from any reliance placed on the content. Where the content includes any translated material, BMJ does not warrant the accuracy and reliability of the translations (including but not limited to local regulations, clinical guidelines, terminology, drug names and drug dosages), and is not responsible for any error and/or omissions arising from translation and adaptation or otherwise.

Open access This is an open access article distributed in accordance with the Creative Commons Attribution Non Commercial (CC BY-NC 4.0) license, which permits others to distribute, remix, adapt, build upon this work non-commercially, and license their derivative works on different terms, provided the original work is properly cited, appropriate credit is given, any changes made indicated, and the use is non-commercial. See: http://creativecommons.org/licenses/by-nc/4.0/.

\section{ORCID iDs}

Shi-sheng Ye http://orcid.org/0000-0003-4306-0460

Tao-Hsin Tung http://orcid.org/0000-0003-2097-8375

\section{REFERENCES}

1 Campbell BCV, Mitchell PJ, Kleinig TJ, et al. Endovascular therapy for ischemic stroke with perfusion-imaging selection. $N$ Engl J Med 2015;372:1009-18.

2 Saver JL, Goyal M, Bonafe A, et al. Stent-retriever thrombectomy after intravenous t-PA vs. t-PA alone in stroke. $N$ Engl J Med 2015;372:2285-95.

3 Goyal M, Demchuk AM, Menon BK, et al. Randomized assessment of rapid endovascular treatment of ischemic stroke. $N$ Engl J Med 2015;372:1019-30.

4 Berkhemer OA, Fransen PSS, Beumer D, et al. A randomized trial of intraarterial treatment for acute ischemic stroke. $N$ Engl J Med 2015;372:11-20.

5 Jovin TG, Chamorro A, Cobo E, et al. Thrombectomy within 8 hours after symptom onset in ischemic stroke. N Engl J Med 2015;372:2296-306.

6 Liu X, Dai Q, Ye R, et al. Endovascular treatment versus standard medical treatment for vertebrobasilar artery occlusion (best): an open-label, randomised controlled trial. Lancet Neurol 2020;19:115-22.

7 Linfante I, Walker GR, Castonguay AC, et al. Predictors of mortality in acute ischemic stroke intervention: analysis of the North American solitaire acute stroke Registry. Stroke 2015;46:2305-8.

8 Flint AC, Cullen SP, Faigeles BS, et al. Predicting long-term outcome after endovascular stroke treatment: the totaled health risks in vascular events score. AJNR Am J Neuroradiol 2010;31:1192-6.

9 Gory B, Mazighi M, Labreuche J, et al. Predictors for mortality after mechanical thrombectomy of acute basilar artery occlusion. Cerebrovasc Dis 2018;45:61-7.

10 Gattringer T, Posekany A, Niederkorn K, et al. Predicting early mortality of acute ischemic stroke. Stroke 2019;50:349-56.

11 Liggins JTP, Yoo AJ, Mishra NK, et al. A score based on age and DWI volume predicts poor outcome following endovascular treatment for acute ischemic stroke. Int J Stroke 2015;10:705-9.

12 Hallevi $\mathrm{H}$, Barreto AD, Liebeskind DS, et al. Identifying patients at high risk for poor outcome after intra-arterial therapy for acute ischemic stroke. Stroke 2009;40:1780-5.

13 Sarraj A, Albright K, Barreto AD, et al. Optimizing prediction scores for poor outcome after intra-arterial therapy in anterior circulation acute ischemic stroke. Stroke 2013;44:3324-30. 
14 Saposnik G, Kapral MK, Liu Y, et al. IScore: a risk score to predict death early after hospitalization for an acute ischemic stroke. Circulation 2011;123:739-49.

15 O'Donnell MJ, Fang J, D'Uva C, et al. The plan score: a bedside prediction rule for death and severe disability following acute ischemic stroke. Arch Intern Med 2012;172:1548-56.

16 Chu X, Yang Y, Zhang F, et al. Validation of iscore and plan score for death in thrombectomy in acute stroke due to anterior circulation large artery occlusion. J Stroke Cerebrovasc Dis 2018;27:3261-5.

17 Powers WJ, Rabinstein AA, Ackerson T, et al. 2018 guidelines for the early management of patients with acute ischemic stroke: a guideline for healthcare professionals from the American heart Association/ American stroke association. Stroke 2018;49:e46-110.

18 von Kummer R, Broderick JP, Campbell BCV, et al. The Heidelberg bleeding classification: classification of bleeding events after ischemic stroke and reperfusion therapy. Stroke 2015;46:2981-6.

19 Zaidat OO, Yoo AJ, Khatri P, et al. Recommendations on angiographic revascularization grading standards for acute ischemic stroke: a consensus statement. Stroke 2013;44:2650-63.
20 Nilanont Y, Komoltri C, Saposnik G, et al. The Canadian neurological scale and the NIHSS: development and validation of a simple conversion model. Cerebrovasc Dis 2010;30:120-6.

21 Linfante I, Starosciak AK, Walker GR, et al. Predictors of poor outcome despite recanalization: a multiple regression analysis of the NASA registry. J Neurointerv Surg 2016;8:224-9.

22 Zaidat OO, Suarez Jl, Santillan C, et al. Response to intra-arterial and combined intravenous and intra-arterial thrombolytic therapy in patients with distal internal carotid artery occlusion. Stroke 2002;33:1821-7.

23 Panni P, Gory B, Xie Y, et al. Acute stroke with large ischemic core treated by thrombectomy. Stroke 2019;50:1164-71.

24 National Institute of Neurological Disorders and Stroke rt-PA Stroke Study Group. Tissue plasminogen activator for acute ischemic stroke. N Engl J Med 1995;333:1581-8.

25 Meyer L, Alexandrou M, Flottmann F et al. Endovascular treatment of very elderly patients aged $\geq 90$ with acute ischemic stroke. $J \mathrm{Am}$ Heart Assoc 2020;9:e014447. 